\title{
Electrochemical Evaluation on Repairs with Mortars in Structures Affected by Chlorides Contamination
}

\author{
M.E. Acosta-Lomelí, * R. Velázquez-González, C. Gaona Tiburcio, \\ F. Almeraya-Calderón, A. Martínez-Villafañe \\ Centro de Investigación en Materiales Avanzados, S.C. \\ División de Deterioro de Materiales e Integridad Estructural / Grupo de Corrosión \\ Miguel de Cervantes 120, Complejo Ind. Chih., C.P. 31109, Chihuahua, Chih. México
}

Received 14 June 2004; accepted in revised form 17 December 2004

\begin{abstract}
Inside the construction industry, in the area of sea water retention structures, the chlorides contamination is a main cause of the corrosion, because in the concrete they could form macro cells upon repairing and applying a mortar free of this ion, forming anodes in the repaired zones, and thus increasing corrosion. In the reinforcements are commonly formed corrosion products because the concrete is exposed to mechanical, physical, chemical or biological conditions that commit and shorten their performance. As a consequence, it is important to determine their useful life and that of the made repairs, using electrochemical techniques that allow determining the level of protection, the effect of the macro cells in the corrosive process of the reinforcement, and the mechanism of deterioration in the structures. The present work was carried out using six beams with preoxided reinforcements, six precracking and one beam blank. After curing, those beams were exposed to accelerated cycles of deterioration for causing the cracking of the concrete, making possible to observe their electrochemical behaviour. The tests carried out were corrosion potential and corrosion rate, through the resistance to the polarization technique.
\end{abstract}

Keywords: corrosion, chlorides, macro cells, electrochemistry, concrete.

\section{Introducción}

Dentro del campo de la construcción, en el área de estructuras de retención de aguas marinas se tiene que una de las causas principales de la corrosión es la contaminación por cloruros, que puede formar macroceldas, si durante una reparación se aplica un mortero libre de este ión, ya que se producen ánodos en las áreas de las zonas reparadas y de esta manera se provoca la corrosión [1], formándose productos de corrosión tales como óxidos voluminosos y expansivos

\footnotetext{
* Corresponding author. E-mail address: www.elena.acosta@cimav.edu.mx
} 
que generan fuerzas de tensión internas. El concreto esta expuesto a condiciones mecánicas, físicas, químicas y biológicas que comprometen y acortan el desempeño de la vida en servicio en las estructuras y por todo lo anterior se considera como el objetivo principal de este trabajo la determinación de la vida útil de la reparación de estructuras, empleando técnicas electroquímicas, determinando el nivel de protección, el efecto en el proceso corrosivo del refuerzo y el mecanismo de deterioro por corrosión en las estructuras, comparando su eficiencia al emplearlos en la práctica (Fig. 1).

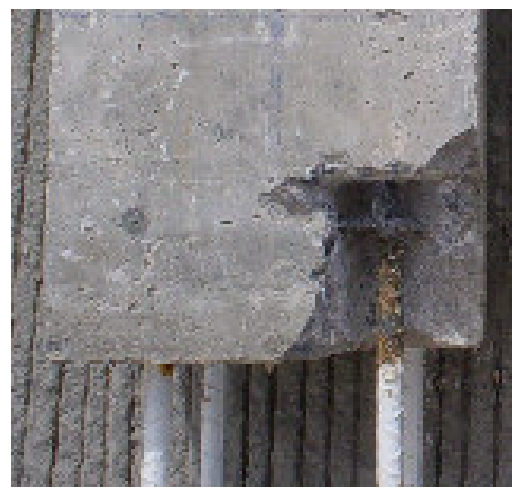

Figura 1. Problema para protección del acero de refuerzo.

Cuando existe algún problema de corrosión, se analiza el daño buscando en la medida de lo posible que la estructura no sea demolida; dependiendo del tamaño del daño y de la importancia estructural, se tiende a emplear medidas alternas como lo es la aplicación de morteros [2], que debe hacerse con control de calidad, sobre todo en la aplicación (Fig.2).

Con la experimentación aquí planteada se busca evaluar el nivel de protección del mortero, así como su efecto en el proceso corrosivo del refuerzo y el mecanismo de deterioro por corrosión, comparando la eficiencia en la práctica del mortero base cemento y base resina epóxica.

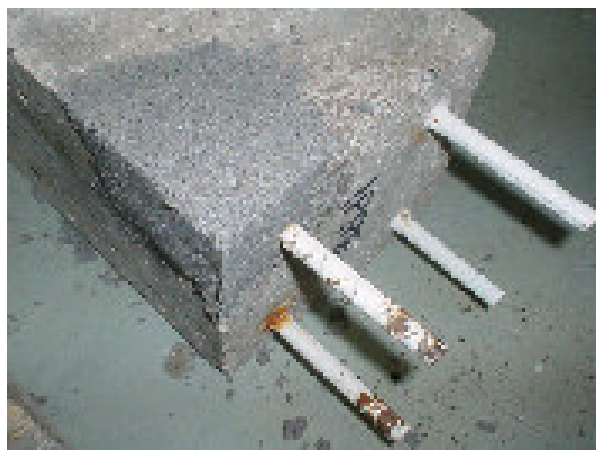

Figura 2. Estructura reparada con mortero.

En este trabajo se realizaron seis especimenes prismáticos con refuerzos limpios, seis con refuerzos preoxidados y un espécimen testigo. Después del curado fueron expuestos a ciclos acelerados de degradación, sometiéndolos a un ambiente agresivo para observar su comportamiento electroquímico. 
Posteriormente a las vigas con refuerzos limpios se les aplicó una carga teórica permisible para lograr un ligero agrietamiento del recubrimiento de concreto, para iniciar así el proceso de corrosión. Las pruebas realizadas fueron: contenido de iones cloruro solubles, medición de potenciales de corrosión, velocidad de corrosión, y observación superficial a través de microscopía electrónica de barrido, para observar el posible daño generado (picaduras) y el análisis elemental de la composición del acero y de los productos de corrosión, empleando para ello el EDAX (análisis de energía dispersiva de RX) del microscopio.

\section{Desarrollo experimental}

Los especimenes, como se observó arriba, fueron 6 vigas preagrietadas, 6 vigas preoxidadas, y 1 viga testigo; de acuerdo a la Norma (C-192 ASTM)[3], las dimensiones de las vigas fueron de 6 x 6 × 20", (Fig. 3 ).

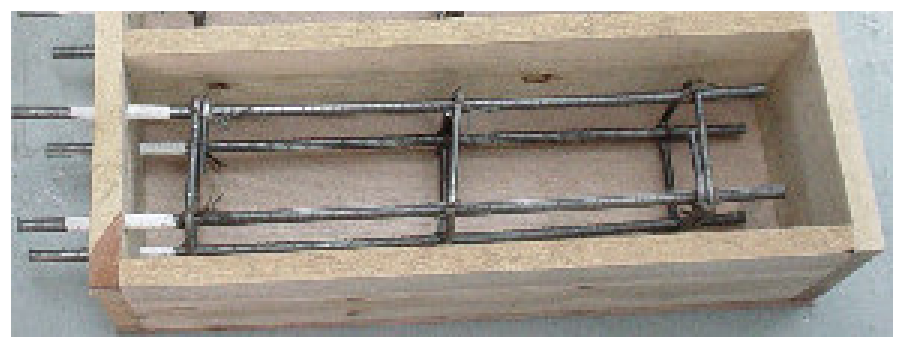

Figura 3. Armado de cimbra para cada una de las vigas.

La elaboración del concreto utilizado fue para una resistencia de f'c $=25 \mathrm{MPa}$, con una relación a/c de 0.58 , el tamaño máximo de agregado de $3 / 4$ ", con acero de refuerzo de $3 / 8$ ".

El acero de refuerzo para las vigas preoxidadas se encintó previamente en algunas secciones quedando así partes expuestas que se pudieron oxidar en salmuera por un período corto de tiempo (Fig. 4).

Posterior a la oxidación las cintas fueron retiradas logrando así tener refuerzos preoxidados en puntos específicos para intentar generar más fácilmente macroceldas (zonas ánodo - cátodo) en los refuerzos.

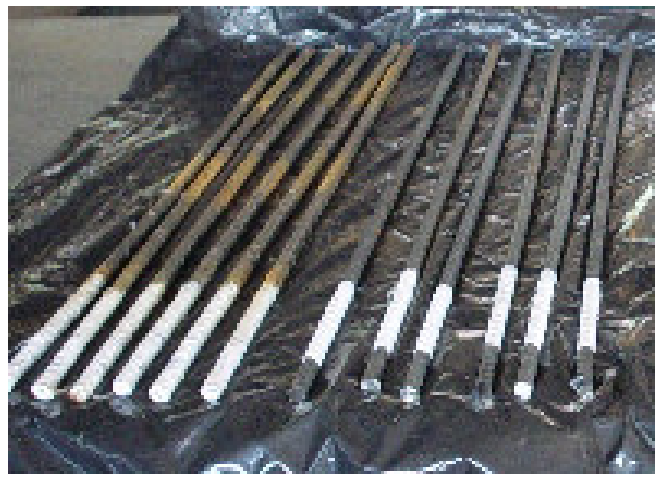

Figura 4. Encintado de secciones del acero de refuerzo. 
También se determinó el contenido de iones cloruro solubles por titulación total, como lo indica la Norma ASTM D-1411[4] al terminar la etapa de curado.

Las vigas preagrietadas estuvieron inmersas en agua simple por un período de 120 días, para posteriormente aplicar la carga en dos puntos centrales y así analizar el comportamiento del espécimen agrietado al quedar expuesto a un ambiente salino. La carga teórica permisible que resiste la sección propuesta para las vigas preagrietadas se calculó con las formulas de la teoría elástica [5], en la cual el acero se carga hasta su límite elástico, o sea, antes de sufrir una deformación permanente (Fig. 5). Estas vigas quedaron expuestas a un ambiente salino al 3.5\% por un período de 217 días (Fig. 6).

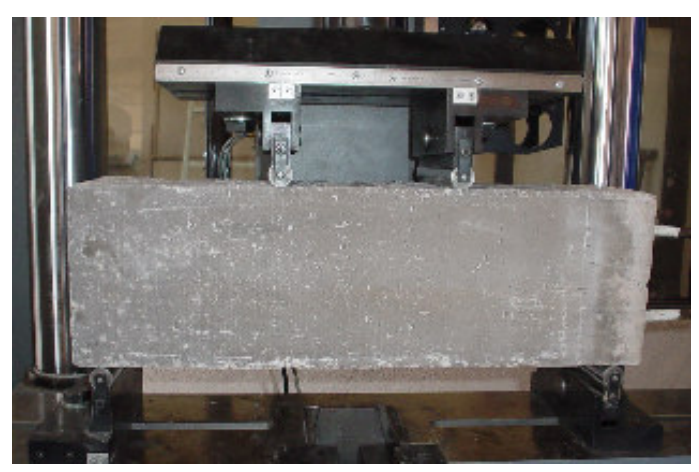

Figura 5. Posición de las cargas sobre las vigas.

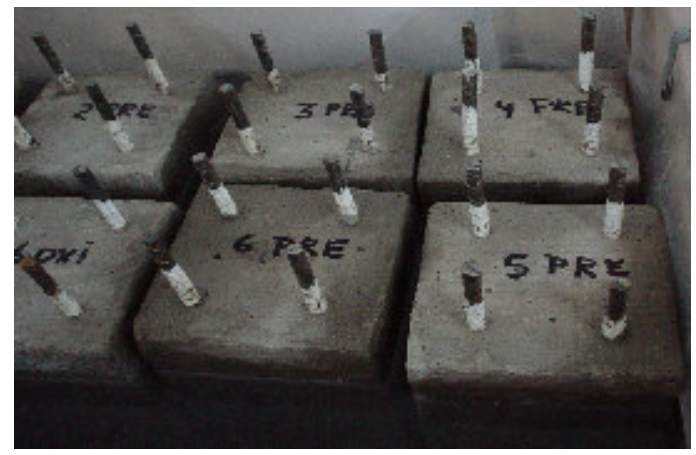

Figura 6. Vigas expuestas a ambiente salino.

Durante el proceso de curado se realizó medición de potenciales a las 13 vigas de concreto, usando un electrodo de referencia de $\mathrm{Cu} / \mathrm{CuSO}_{4}$ y un voltímetro digital de acuerdo a la Norma ASTM C - 876-91 [6], utilizando las mismas varillas de refuerzo como electrodo de trabajo, (Fig. 7). 


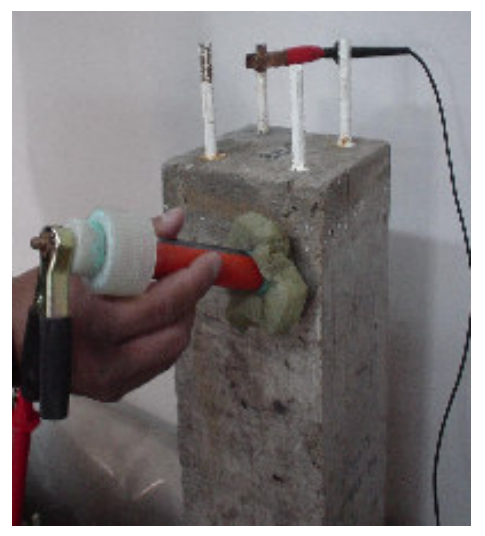

Figura 7. Medición de potenciales.

Para la medición de velocidad de corrosión se empleó la técnica de resistencia a la polarización $(\mathrm{Rp} \pm 20 \mathrm{mV})$ [7], utilizando para ello un equipo de corrosión electroquímica "Potenciostato/galvanostato", que emplea un censor con anillo de guarda de acero inoxidable [8] y un electrodo de referencia de $\mathrm{Cu} / \mathrm{CuSO}_{4}$ (Fig. 8).

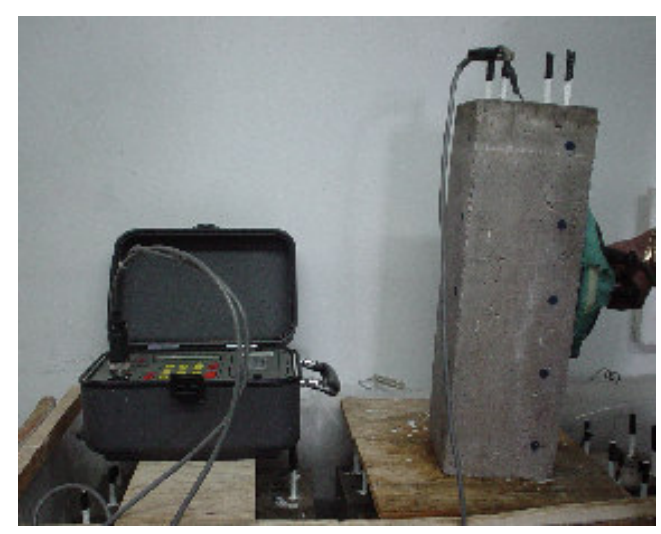

Figura 8. Equipo de corrosión Gecor 6 y censores de corrosión.

\section{Resultados}

La determinación de iones cloruro del concreto natural inicialmente fue de $0.04314 \%$, siendo insignificante. Una vez terminado el período exposición al ambiente salino fue de $0.88395 \%$ en las vigas preagrietadas y preoxidadas siendo el riesgo posible a corroerse (ver Tabla 1) aplicándose el criterio de riesgo de corrosión de la barra de refuerzo con relación a los rangos de $\mathrm{C}_{\text {crit }}[9]$. 
Tabla 1. El riesgo de corrosión de la barra de refuerzo con relación a los rangos de $\mathrm{C}_{\text {CRIT }}[10]$.

\begin{tabular}{cc}
\hline $\begin{array}{c}\text { Concentración del cloruro a la profundidad del } \\
\text { acero (\% por peso del cemento) }\end{array}$ & $\begin{array}{c}\text { Riesgo para la iniciación de la } \\
\text { corrosión }\end{array}$ \\
\hline$<0.4 \%$ & Insignificante \\
$0.4-1.0$ & Posible \\
$1.0-2.0$ & Probable \\
$>2.0$ & Seguro \\
\hline
\end{tabular}

La medición de potenciales de corrosión en el proceso de curado para las vigas preagrietadas varió entre -160 y $-240 \mathrm{mV}$ (Gráfica 1), donde se aprecia que los potenciales de menos de $-200 \mathrm{mV}$ se detectan en las vigas 1 a 3 .

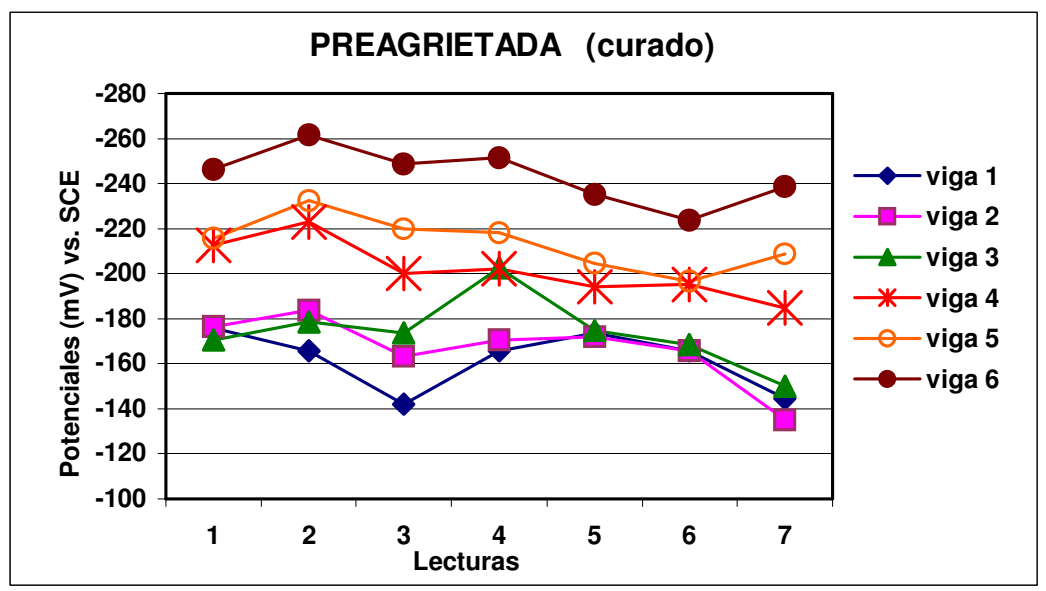

Gráfica 1. Potenciales promedio de 6 vigas precargadas en el proceso de curado.

Para las vigas preoxidadas en el proceso de curado el potencial se encontró entre $-150 \mathrm{y}-250 \mathrm{mV}$ aproximadamente entre las 6 vigas (Gráfica 2).

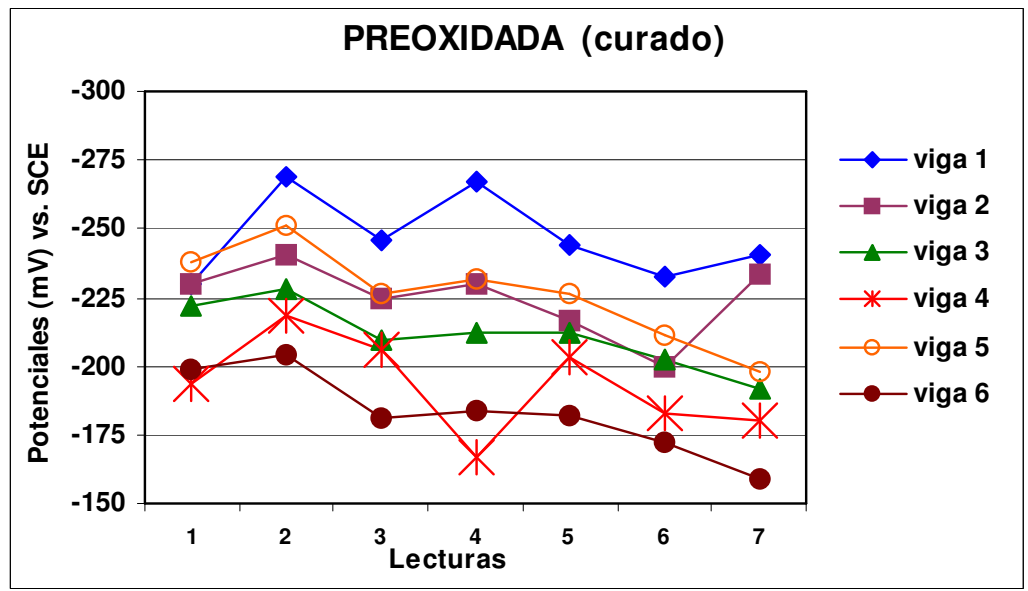

Gráfica 2. Potenciales promedio de las 6 vigas preoxidadas en el proceso de curado. 
Una vez pasado el proceso de curado la medición de potenciales para las vigas preagrietadas fue cambiando de -100 a $-650 \mathrm{mV}$, para todos los especimenes (Gráfica 3).

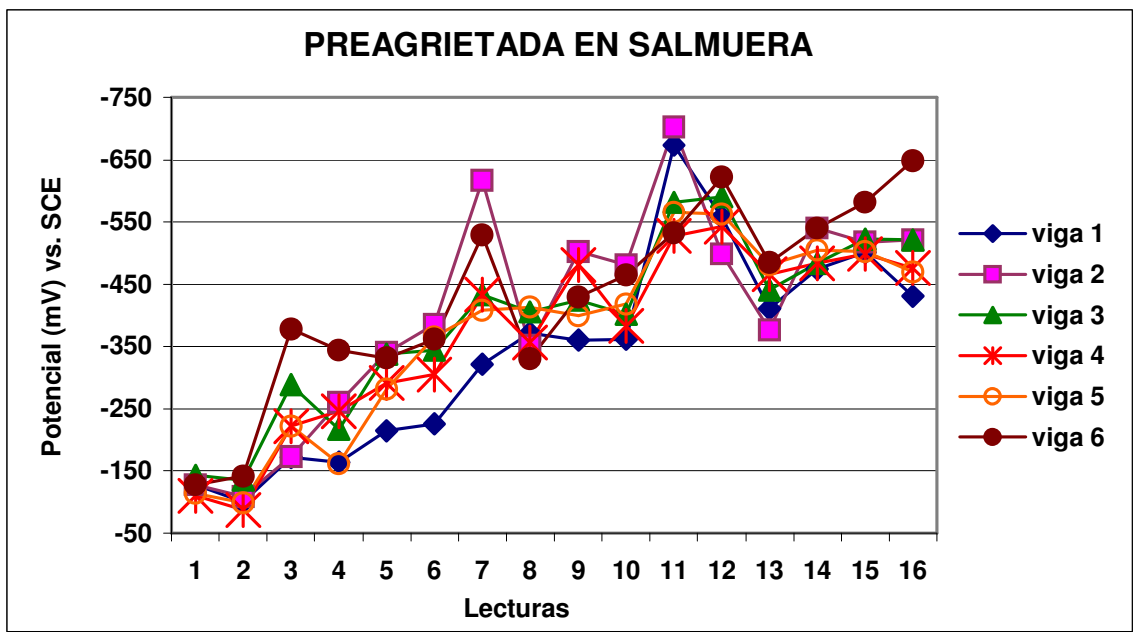

Gráfica 3. Potenciales promedio de las 6 vigas preagrietadas expuestas en salmuera.

Y la medición de potenciales para las vigas preoxidadas osciló entre -350 y $600 \mathrm{mV}$ (Gráfica 4), observando una variación en el tiempo más pausada que en el caso de los especimenes preagrietados.

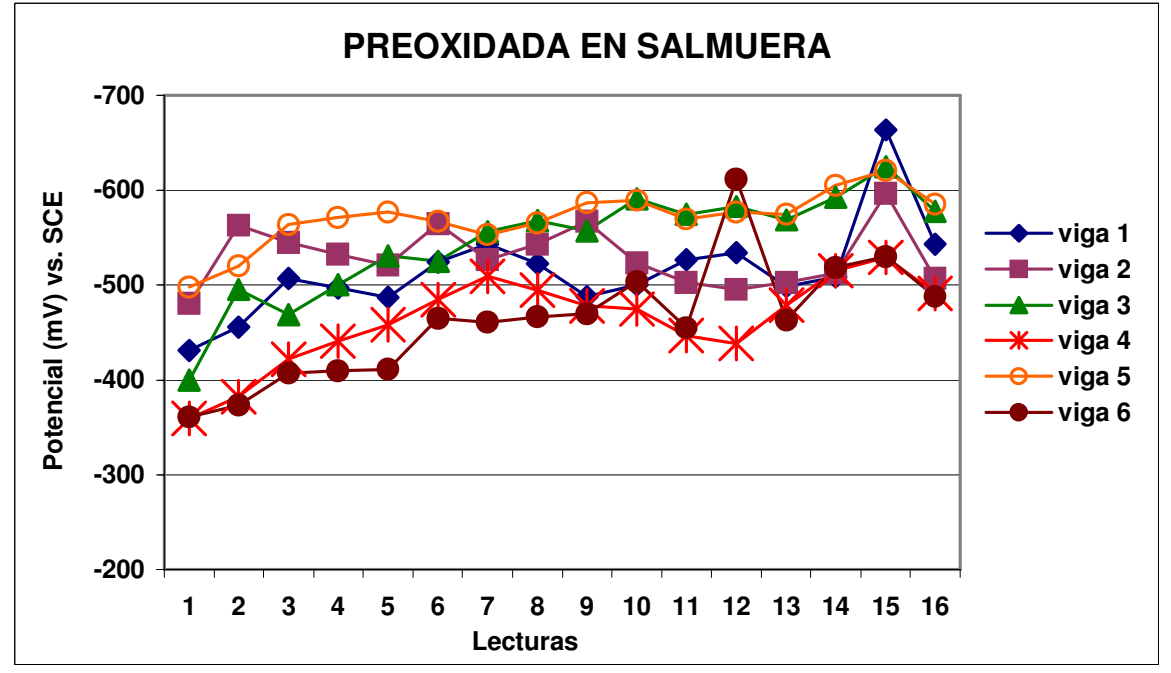

Gráfica 4. Potenciales promedio de las 6 vigas preoxidadas en salmuera.

Basándose en los criterios de clasificación de $\mathrm{I}_{\text {corr }}$ en términos de vida útil de la red Durar [11] se analizan velocidades de corrosión obtenidas en los dos tipos de especimenes (Tabla 2). 
Tabla 2. Velocidad de corrosión en términos de vida útil.

\begin{tabular}{|c|c|c|c|c|}
\hline \multirow{2}{*}{ VIGA } & \multicolumn{2}{|c|}{ Velocidad de corrosión $/\left(\mu \mathrm{A} / \mathrm{cm}^{2}\right)$} & \multicolumn{2}{c|}{ Vida útil (años) } \\
\cline { 2 - 5 } & Preagrietada & Preoxidada & Preagrietada & Preoxidada \\
\hline $\mathbf{1}$ & 0.021 & 0.290 & 80 a 100 & $60-80$ \\
\hline $\mathbf{2}$ & 0.362 & 0.379 & 60 a 80 & $60-80$ \\
\hline $\mathbf{3}$ & 0.487 & 0.558 & 60 a 80 & $40-60$ \\
\hline $\mathbf{4}$ & 0.310 & 0.529 & 60 a 80 & $40-60$ \\
\hline $\mathbf{5}$ & 0.349 & 0.575 & 60 a 80 & $40-60$ \\
\hline $\mathbf{6}$ & 0.561 & 0.412 & 60 & $60-80$ \\
\hline
\end{tabular}

\begin{tabular}{|c|c|c|c|c|}
\hline \multirow{2}{*}{ VIGA } & \multicolumn{2}{|c|}{ Velocidad de corrosión $/\left(\mu \mathrm{A} / \mathrm{cm}^{2}\right)$} & \multicolumn{2}{c|}{ Vida útil } \\
\cline { 2 - 5 } & Preagrietada & Preoxidada & Preagrietada & Preoxidada \\
\hline $\mathbf{1}$ & 0.021 & 0.290 & Despreciable & Baja \\
\hline $\mathbf{2}$ & 0.362 & 0.379 & Baja & Baja \\
\hline $\mathbf{3}$ & 0.487 & 0.558 & Baja & Moderada \\
\hline $\mathbf{4}$ & 0.310 & 0.529 & Baja & Moderada \\
\hline $\mathbf{5}$ & 0.349 & 0.575 & Baja & Moderada \\
\hline $\mathbf{6}$ & 0.561 & 0.412 & Baja a moderada & Baja \\
\hline
\end{tabular}

\section{Conclusiones}

En cuanto a la determinación de iones cloruro, basándose en el criterio de riesgo de corrosión, se tiene que existe en las vigas preoxidadas un posible riesgo a corroerse. En la medición de potenciales, las vigas precargadas la tendencia fue muy baja inicialmente, y posterior a la aplicación de la precarga la tendencia fue mayor, indicando que el acero se esta corroyendo. En la velocidad de corrosión basada en la clasificación de Icorr en términos de vida útil, se tiene que el nivel de corrosión es moderado tanto en las vigas precargadas como en las preoxidadas. En el periodo de exposición considerado, su comportamiento es muy similar entre unas vigas y otras, ya que existe poca concentración de cloruros, principalmente en la pared externa del concreto de las vigas. Como el concreto presenta características de buena calidad, dando un mantenimiento adecuado para protección del acero de refuerzo de las vigas, estas pueden alcanzar la vida útil para la cual fueron diseñadas.

\section{Reconocimientos}

El autor agradece al Cimav por su apoyo, a Conacyt por la beca otorgada y a Coprechisa por la donación de los materiales. 


\section{Evaluacion Electroquimica de Reparaciónes con Morteros en Estructuras Afectadas por Contaminación de Cloruros}

\section{Resumen}

Dentro del campo de la construcción, en el área de estructuras para retención de aguas marinas, la contaminación por cloruros es una causa principal de la corrosión, se pueden formar macroceldas al reparar y aplicar un mortero libre de este ión, produciéndose ánodos en las áreas de las zonas reparadas, y provocando con ello la corrosión. En el acero de refuerzo se forman productos de corrosión comúnmente porque el concreto esta expuesto a condiciones mecánicas, físicas, químicas o biológicas que comprometen y acortan su desempeño, por ello es importante determinar su vida útil y la de las reparaciones efectuadas, empleando técnicas electroquímicas que permitan determinar el nivel de protección, el efecto de las macroceldas en el proceso corrosivo del refuerzo, y el mecanismo de deterioro en las estructuras. En el presente trabajo se realizaron seis vigas preagrietadas, seis preoxidadas y una viga testigo, después del curado se expusieron a ciclos acelerados de deterioro para provocar el agrietamiento del concreto y observar su comportamiento electroquímico previo y posterior a su reparación. Las pruebas realizadas son: medición de potenciales de corrosión y velocidad de corrosión, empleando la técnica de resistencia a la polarización.

Palabras clave: corrosión, cloruros, macroceldas, electroquímica, concreto.

\section{Referencias}

1. P.C. Borges "Corrosión en estructuras de concreto armado" Teoría, inspección, diagnóstico, vida útil y reparaciones, IMCYC, México, D.F. Primera edición (1998).

2. G.W. Read, Ir. Curtis, E. Zimmer, and G.R. Hall "Cementos y Morteros" Sauereisen Cements Company, Pittsburgh, Pennsylvania (1999)

3. ASTM C 192/ C 192M- 98 "Standard Test Methods for Production and Cured Specimens of Test Concretes in the Laboratory" (1998).

4. ASTM D 1411-99 "Standard Test Methods for Water-Soluble Chlorides Present as Admixtures in Graded Aggregate Road Mixes" (1999).

5. Cálculo Estructural I, Facultad de Ingeniería, Universidad Autónoma de Chihuahua (1999).

6. ASTM C-876-91 "Standard Test Method for Half-Cell Potentials of Uncoated Reinforcing Steel in Concrete", American Society for Testing and Materials, Philadelphia, USA (1991).

7. S. Feliu, J.A. Gonzalez and C. Andrade, "Electrochemical Methods for OnSite Determinations of Corrosión Rates of Rebars", Techniques to Asses the Corrosion Activity of Steel Reinforced Concrete Structures, ASTM STP 1276, Neal S. Berque, Eduard Escalante, Charles K. Nmai and David Whiting, Eds., American Society for Testing and Materiales, (1996).

8. S. Feliu, J.A. Gonzalez, S.J.R. Feliu, M.L. Escudero, Y. Maribona, V. Austin, C. Andrade, J.A. Bolaño, F. Jimenez, "Corrosion Detecting Probes for use with a Corrosion Rate Meter for Electrochemically Determining the 
Corrosion Rate of Reinforced Concrete Structures", U.S. Patent No. 5.259.944 (1993)

9. A.A.A. Torres, M.M. Martínez, "Diseño de Estructuras de Concreto con Criterios de Durabilidad", Secretaría de Comunicaciones y Transportes, Instituto Mexicano del Transporte, Publicación Técnica $\mathrm{N}^{\circ} 181$ ISSN 0188-7297, Sanfandíla, Qro. México (2001).

10. P. Bamforth, "Chloride penetration and service life", Corrosion and protection of metals in contact with concrete - COST-509 Workshop, Sevilla, España, Septiembre (1995).

11. R.O. Troconis, DURAR "Manual de inspección y diagnostico de corrosión en estructuras de hormigón armado", CYTED, (Programa Iberoamericano de Ciencia y Tecnología para el Desarrollo), Op.Cit. Julio (1998). 